\title{
ESPACIOS DE PARTICIPACIÓN SOCIAL Y SALUD EN LA VEJEZ FEMENINA
}

\author{
Deisy Krzemien ${ }^{l}$ \\ Enrique Lombardo ${ }^{2}$
}

\section{Resumen}

El presente artículo propone estudiar la correlación entre participación social y salud en la vejez femenina. Mediante un diseño correlacional descriptivo de carácter trasversal, se administró un cuestionario estructurado en el marco de una entrevista semiestructurada a una muestra de 70 sujetos de sexo femenino de 60 a 80 años de edad, de la ciudad de Mar del Plata. Se evaluó el estado de salud físico y mental y la participación en actividades y relaciones sociales valoradas social y subjetivamente. Se consideró la frecuencia de asistencia, la satisfacción y la valoración subjetiva de la participación social. Proponemos el concepto de espacios de participación social significativa (EPSS) el cual integra la oferta institucional, actividades valoradas social y subjetivamente, y relaciones sociales significativas, como componentes interdependientes. Se realizó un análisis cuantitativo de los datos. Los resultados mostraron una correlación positiva y estadísticamente significativa entre participación social significativa y salud en adultas mayores $(r=0.633, p=0.01)$. Las mujeres ancianas que no participan en actividades y relaciones sociales evidencian un estado de salud psíquica significativamente menor que aquellas quienes muestran un estilo de vida socialmente activo. La participación social significativa promueve un modelo de envejecimiento saludable.

Palabras-clave: Vejez femenina. Participación social significativa. Salud.

\footnotetext{
${ }^{1}$ Licenciada en Psicología, Becaria de Investigación y docente de Psicología Evolutiva y Filosofía del Hombre, Facultad de Psicología, Universidad Nacional de Mar del Plata. E-mail: dekrzem@mdp.edu.ar

2 Licenciado en Psicología, investigador y docente de Psicología del Desarrollo, Facultad de Psicología, Universidad Nacional de Mar del Plata. E-mail: elombar@sinectis.com.ar
} 


\section{Introducción}

La conceptualización de la salud se torna una misión problemática debido, por un lado, a que se trata de un concepto de contenido valorativo en gran medida; y por otro, porque la evaluación de los síntomas y procesos físicos, psíquicos y comportamentales que se utilizan para designar una persona como sana o enferma, varían según las representaciones sociales y paradigmas científicos dominantes en cada cultura y momento histórico.

En los últimos años se han producido modificaciones conceptuales importantes en relación a la salud, favoreciendo la introducción de factores psicosociales y comunitarios en función de un enfoque basado en la salud y no meramente en la ausencia de enfermedad (BARO, 1985). La definición de salud de la Organización Mundial de la Salud alude al "[ . . . ] estado de bienestar físico, mental y social, y no sólo la ausencia de enfermedad." (1948, p. 2). La importancia de esta concepción radica en la consideración de una medida positiva de salud y la inclusión de la percepción subjetiva de bienestar personal (LA ROSA,1988). En otros términos, la salud sería la capacidad de desarrollar el potencial personal y responder en forma positiva a las exigencias del medio, destacando recursos psicosociales y capacidades físicas (PINEAULT; DAVELUY, 1995). Desde este enfoque de la promoción de la salud, esta resulta dependiente no sólo del componente biológico, sino de las condiciones del medio relacional en sentido amplio, y se halla vinculada a un cierto estilo de vida (DULCEY-RUIZ, 1988; EPP et al., 1996).

Cabe destacar un hito importante en la definición de la salud representado por la Carta de Ottawa para la Promoción de la Salud (ORGANIZACIÓN MUNDIAL DE LA SALUD, 1986) presentada a los organismos internacionales de la salud, donde se exponen, de una manera integrativa, los múltiples factores que afectan el estado de salud de un individuo o población: este concepto contempla la descomposición del campo de la salud en cuatro amplios componentes: biología humana, medio ambiente, estilo de vida y organización de la atención de la salud (LALONDE, 1986).

La convergencia de estos factores determina el estado de salud de un sujeto (Figura 1). 


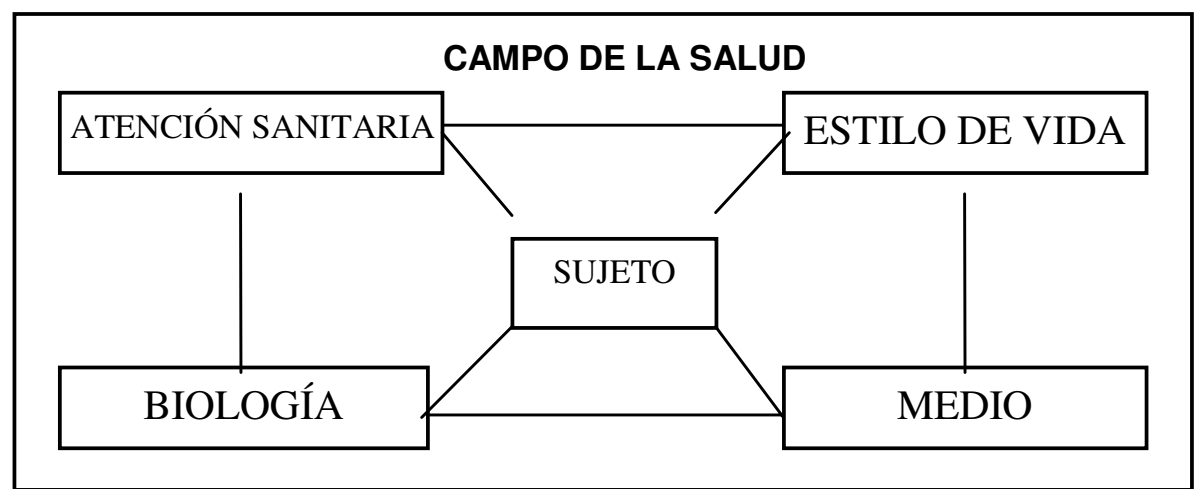

FIGURA 1: Componentes del campo de la salud. (OMS, 1986)

En el caso de la vejez, nos enfrentamos hoy en día con la carencia de modelos integradores que brinden un patrón de referencia específico para el abordaje de la salud. La inserción social del adulto mayor no alcanza actualmente niveles esperables para una integración social adecuada (NACIONES UNIDAS, 1982), hecho ligado, a nuestro entender, en buena medida, a la falta de significatividad de la participación social y al predominio de representaciones sociales negativas vinculadas al envejecimiento. En este sentido, entendemos necesario una redefinición de la participación del adulto mayor en el ámbito social.

Alcanzada la etapa final de la vida, el medio social en general presupone que el adulto mayor es incapaz de realizar actividades de acuerdo a la valoración negativa del proceso de envejecimiento, concibiéndolo como ligado al deterioro progresivo de las capacidades funcionales (BARROS LAZAETA, 1989). Esta condición contribuye a inhabilitarlo como sujeto de acción, a restarle sentido a la vida y a centrar su atención en sí mismo y en sus quejas somatopsíquicas.

La tradición en estudios sobre la vejez denuncia una centración en la vulnerabilidad del cuerpo viejo, en los cambios sociales que implican abandono de roles, y en los duelos y pérdidas como característicos de esta etapa vital, descalificando al adulto mayor como sujeto de acción, saludable, participante y social. Esta concepción encuentra su basamento ideológico en la Teoría del Desapego (CUMMINGS; HENRY, 1961). Por su parte, la sociedad, en muchos casos, ofrece escasas posibilidades de una vida activa. En el caso de las mujeres ancianas, la participación no sólo tiene que ver con la iniciativa que podría vincularse a variables individuales sino con el lugar que la sociedad asigna 
a la mujer que envejece. Es decir, hay creencias compartidas en el orden social, diferentes según las culturas, que ofrecen distintos roles sociales a la mujer. Las mujeres ancianas son las más vulnerables desde el punto de vista de la salud. Si bien las mujeres presentan mayor esperanza de vida con respecto a los hombres, sufren una doble prejuiciación: como mujeres y como viejas (ODDONE, 1990; PÉREZ-ANDRÉS, 1993). Así, se suma a la exclusión social proveniente del género, el predominio de connotaciones sociales negativas atribuidas al envejecimiento.

Si bien las personas que envejecen experimentan un gradual desgaste del funcionamiento del organismo, esto no implicaría necesariamente un proceso degenerativo ni involución en términos de salud (BARROS LAZAETA, 1989). Siguiendo los postulados de la Teoría de la Actividad (HAVIGHURST, 1963), la declinación de las habilidades se debe más a la falta de actividad y al aislamiento social que al proceso de envejecimiento propiamente dicho (LOWENTHAL; ROBINSON, 1976).

En un intento de estudiar las consecuencias que tiene el grado de participación social del adulto mayor sobre el estado de salud, es necesario considerar una doble vía: social e individual. Por vía social, la representación social negativa de la vejez supone una baja expectativa de participación para el anciano, lo cual se evidencia en una concentración de la atención social de la vejez en el ámbito sanitario y en la inadecuación y/o carencia de ofertas de participación en el medio social. Desde la vía individual, la interiorización de esos estereotipos sociales negativos de la vejez por parte del sujeto disminuye también la expectativa personal y la valoración subjetiva de participación social. Esto se expresa en conductas como autoexclusión, descalificación personal, desinterés por actividades y retracción personal. Por ambas vías, social e individual, se contribuye a una ausencia de participación social en la vejez, lo cual calificaría un estilo de vida caracterizado por el aislamiento social y en términos de salud, supone un deterioro del estado de salud tanto objetivo como percibido subjetivamente. Más aun, esta imagen de la vejez no hace más que reafirmar el estereotipo negativo que asocia vejez y enfermedad (Figura 2).

Actualmente se considera a la "participación social" como un valor del propio campo de la salud, distinguiéndose la pertenencia a un grupo social y un estilo de vida activo como vinculados al mantenimiento de una buena salud y a 


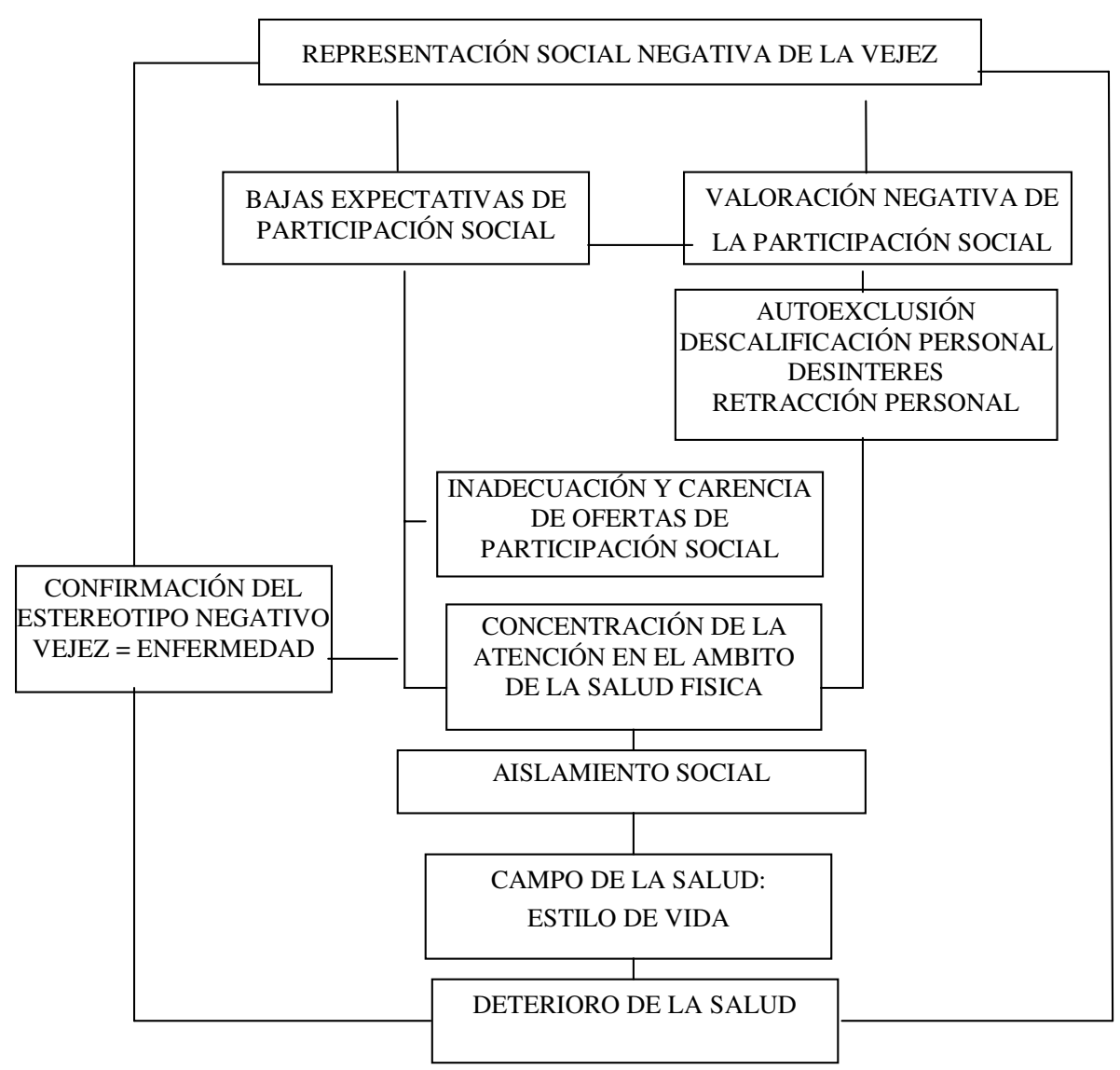

FIGURA 2: Modelo de la relación entre el aislamiento social y el deterioro de la salud en la vejez

la prevención de enfermedades (EPP et al., 1996).

Investigaciones de la Organización Mundial de la Salud (PSZEMIAROWER, 1988) demuestran que las personas que participan en grupos sociales mantienen un estado de salud superior a los que están socialmente aislados, y que una vida rica en relaciones afectivas significativas tiende a prolongarse. Los estudios de Cassel (1974) atribuyen a las relaciones sociales próximas funciones protectoras contra la enfermedad. El conocido trabajo de Berkman y Syme (1979) muestra que los sujetos ancianos que informaron mayor grado de contactos sociales sobrevivieron más tiempo que aquellos que informaron menos recursos sociales. Estos datos son congruentes con los hallados por otras investigaciones (FERNÁNDEZ-BALLESTEROS, 1992). 
También los estudios de Moos y Lembe (1985), entre otros, han contribuido a identificar las consecuencias positivas para la salud en la vejez del mantenimiento de relaciones sociales. Otros estudios en esta línea hacen referencia al estilo de vida participativo versus el aislamiento y sedentarismo, más allá de las determinaciones biológicas u orgánicas del individuo: Thonse (1985) ha mostrado altas correlaciones entre percepción subjetiva de bienestar y estilo de vida activo para enfrentar las dificultades; en contraste con altas correlaciones entre percepción subjetiva de malestar y un estilo de vida pasivo ante las dificultades. También investigaciones (THIED AGE LEARNING INTERNATIONAL STUDIES, 1998) demostraron la importancia de la educación - en tanto actividad psicosocial - para una mejor calidad de vida en la vejez. Existe evidencia empírica (BROADHEAD et al., 1983; BERKMAN, 1984; HAREL; DEIMLONG, 1984; WARD, 1985; MUCHINIK, 1990; BOWLING; BROWNE, 1991; BELLO JUJÁN et al., 1999) sobre la importancia de las "redes de apoyo social"3 para el bienestar de la población anciana. Recientes investigaciones (MATEOS ALVAREZ et al., 2001; SEIDMANN, 2001) destacan la relación entre el apoyo social y la disminución de factores de riesgo de la salud en la vejez.

Los vínculos del anciano con la familia y el medio social son percibidos desde la valoración singular. Al estudiar la inserción social del adulto mayor y el papel de las relaciones sociales en la salud del anciano, es importante considerar como él mismo percibe dichas relaciones.

De acuerdo a estas ideas, proponemos algunos conceptos que podrían representar un modelo para pensar la salud en la vejez, el cual denominamos "espacios de participación social significativa" (EPSS). Dicho concepto permite integrar la oferta de espacios institucionales, actividades valoradas social y subjetivamente, y relaciones sociales significativas, como componentes interdependientes que conjuntamente promueven la salud en la vejez, como se representa en la figura 3 .

\footnotetext{
La red social de apoyo se refiere a entornos humanos que operen como red de sostén en situación de crisis y siempre que sea percibida como un beneficio para quien la recibe. En términos operativos, se refiere a la ayuda, orientación, información, cuidado, que un sujeto recibe o percibe recibir de su entorno social (DABAS, 1993).
} 


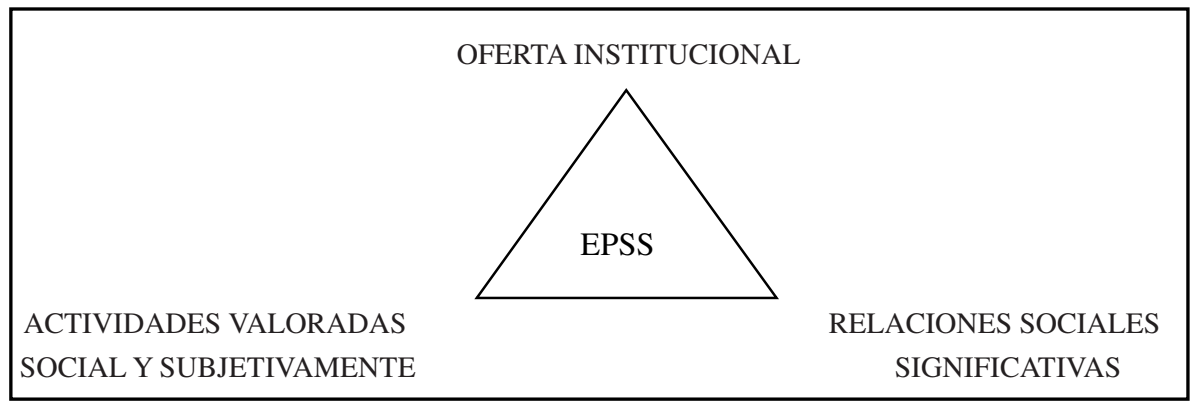

FIGURA 3: Componentes básicos de los espacios de participación social significativa para una vejez saludable

Definimos como espacios de participación significativa a aquellos ámbitos extrafamiliares que proponen actividades para el adulto mayor, caracterizados por el establecimiento de relaciones sociales y donde se obtiene una retribución personal de diversa índole (formación educativa, servicios sanitarios, apoyo social, emocional o material, remuneración económica, etc.), siendo percibidos subjetivamente como beneficiosos. La oferta institucional de espacios de participación del adulto mayor está sostenido por las prácticas sociales legitimadas a través de instituciones de variado tipo, abarcando las áreas de educación, laboral-ocupacional, actividad física, esparcimiento, artística, socio-comunitaria, religioso-espiritual. Las relaciones sociales significativas se definen como el proceso de integración e interacción social percibido subjetivamente como beneficioso, considerando la naturaleza, frecuencia y satisfacción de las relaciones sociales. Por último, las actividades valoradas suponen tomar parte en forma activa y comprometida en tareas valoradas social y subjetivamente.

Con el propósito de estudiar la relación entre la participación social significativa y la salud física y mental en la vejez femenina consideraremos los datos empíricos de un estudio desarrollado en el marco del Grupo de Investigación Temas de Psicología del Desarrollo de la Facultad de Psicología de la Universidad Nacional de Mar del Plata, Argentina.

\section{Métodos}

\subsection{Sujetos}

Se conformó una muestra no probabilística de 70 sujetos del sexo femenino de 60 a 80 años de edad, seleccionados intencionalmente según los 
siguientes criterios de inclusión y exclusión - a fin de guardar homogeneidad de la muestra y evitar el efecto de variables espúreas - sujetos exentos de patologías de invalidez física o cognitiva, de nivel socioeconómico medio, con estudios primarios completos y residentes de cinco años como mínimo de la localidad marplatense. Se excluyeron sujetos que hubiesen enviudado o perdido un familiar cercano en los últimos dos años para eludir la incidencia de esta variable sobre los puntajes que se obtuvieran en las medidas de salud mental.

La muestra procede de lugares públicos e instituciones de la cuidad que proponen actividades de variada índole para la tercera edad.

\subsection{Diseño}

Estudio correlacional descriptivo de carácter trasversal.

\subsection{Procedimiento}

Se procedió a un estudio piloto con el fin de determinar la aptitud de los procedimientos metodológicos y del instrumento para abordar la realidad. Para ello, se seleccionaron intencionalmente 25 ancianas de 60 a 80 años de edad según lo criterios mencionados. Todas las sujetos prestaron su consentimiento voluntario. Se administró el cuestionario en el marco de una entrevista semiestructurada. El estudio piloto arrojó los siguientes resultados: disposición e interés por el objeto de estudio en las entrevistadas, buena recepción del cuestionario (en cuanto a temáticas que aborda, grado de intimidad de las preguntas, extensión y profundidad de los ítems), se evidenció una tendencia a la adaptabilidad del instrumento en cuanto a su calidad y adecuación a los atributos que se pretendían medir, y se registró la necesidad de ciertos ajustes en cuanto a algunos de los ítems.

Se realizó el estudio definitivo entre junio y setiembre de 1999, mediante la autorización y financiación de la Secretaría de Investigación y Postgrado de la Universidad Nacional de Mar del Plata y la Secretaría de Calidad de Vida de la Municipalidad del Partido de General Pueyrredon.

Los sujetos del estudio fueron informados acerca de los objetivos del proyecto de investigación, de la importancia de su participación, del carácter confidencial de los datos aportados, y de la disposición de los resultados una vez elaborado el informe de investigación. Los sujetos debieron expresar en 
forma escrita su consentimiento de participación en el mismo a la hora de responder al cuestionario. La administración fue realizada en forma individual por parte de los mismos investigadores, en una sesión de 50 minutos: 15 minutos para Sección I (salud) y 25 para Sección II (participación social significativa), con un intervalo de descanso de 10 minutos entre ambas secciones, lo cual se convino previamente con los sujetos del estudio.

Para el análisis de los datos, se utilizaron las pruebas estadísticas descriptivas habituales y de inferencia ( $r$ de Pearson). El paquete estadístico utilizado fue el SPSS entorno Windows.

\subsection{Materiales}

La recolección de datos se llevó a cabo mediante un cuestionario estructurado constituido por dos partes: La Sección I del cuestionario evalúa el estado de salud y consta de 42 ítems, agrupados en dos escalas independientes. La Sección A se refiere a la escala salud física, entendida como el adecuado funcionamiento del cuerpo, resistencia a enfermedades y percepción subjetiva de que los órganos, funciones y capacidades físicas responden apropiadamente. Esta escala está estructurada en 27 ítems (diagnóstico clínico de presencia de enfermedades, sentimiento de malestar, estar en tratamiento médico, intervenciones quirúrgicas, alcoholismo, tabaquismo, pérdida de función u órgano, debilidad física, susceptibilidad a infecciones, dolor, medidas de prevención, consumo de fármacos, entre otros). La Sección $B$ se refiere a la escala de salud mental, entendida como la capacidad funcional del organismo, adaptación al medio físico y social, habilidad de mantener relaciones sociales armoniosas y percepción subjetiva de bienestar. Esta escala está estructurada en 15 ítems (ansiedad, angustia, situación de duelo, insomnio, depresión, temores irracionales, tensión, sentimiento de pérdida, concentración cognitiva, memoria, productividad, entre otros). Estos ítems expresan conductas y estados que el sujeto refiere en relación a la salud, los cuales están teóricamente basados en el cuestionario Instrumento de Evaluación de la Calidad de Vida y la Salud - WHOQOL-100 - (OMS, 1998). Para los ítems de salud mental se utilizaron los indicadores de la clasificación internacional de problemas de salud. (CISAP-2, 1996; COMITÉ DE CLASIFICACIÓN DE LA WORLD..., 1998). Los ítems son dicotómicos según una escala de 1-2 puntos donde 1 corresponde a "mala 
salud" y 2 a "buena salud". La suma de las puntuaciones de ambas dimensiones (salud física y salud mental) proporciona un índice global de salud; sus valores posibles oscilan entre 50 (mala salud en todos los ítems) y 90 puntos (buena salud en todos los ítems). El cuestionario evalúa el estado de salud de acuerdo a una escala de 4 niveles: 1 - bajo, 2 - medio-bajo, 3 - medio-alto, y 4 - alto. La Sección II del cuestionario evalúa la participación social significativa mediante 87 ítems agrupados en dos escalas. La escala $A$ está referida a las relaciones sociales familiares y extrafamiliares, considerando la naturaleza, frecuencia y satisfacción subjetiva de la relación. Está conformada por 24 ítems (convivencia, calidad y satisfacción de la relación, apoyo social, expresión de emociones, sentimiento de soledad, personas significativas). La escala $B$ evalúa las actividades sociales que abarcan las áreas de educación, laboral-ocupacional, actividad física, esparcimiento, artística, sociocomunitaria, religioso-espiritual, considerando la frecuencia y satisfacción de la participación. Esta escala se compone por 63 ítems (concurrencia a curso, taller, grupo de lectura, de reflexión, de autoayuda, club, clase de gimnasia, programa deportivo, iglesia, reuniones sociales, actividades voluntarias, laborales, viajes). Los ítems son dicotómicos según una escala de 1-2 puntos donde 1 corresponde a "sin participación" y 2 a "con participación”. Para la evaluación de la frecuencia en la concurrencia se utilizó una escala de tres valores: raramente, a veces y casi siempre, y también para la consideración del grado de satisfacción: poco, más o menos y mucho. La suma de las puntuaciones de ambas dimensiones (relaciones sociales y actividades) proporciona un índice global de participación social significativa (PSS), cuyos valores posibles oscilan entre 15 (nula PSS en todos los ítems) y 225 puntos (óptima PSS en todos los ítems). El cuestionario evalúa la calidad y cantidad de pss de acuerdo a una escala de 5 niveles: 1 - bajo, 2 - medio-bajo, 3 - medio, 4 - medio-alto, y 5 - alto.

\section{Resultados}

La evaluación del estado de salud de la muestra revela que los sujetos alcanzan una puntuación entre un mínimo de 58 puntos y un máximo de 86 puntos, siendo la media aritmética de 71.32 puntos. En cuanto a la participación social significativa, los sujetos alcanzan una puntuación entre los 15 y los 199 puntos, siendo la media del grupo de 84.40 . 


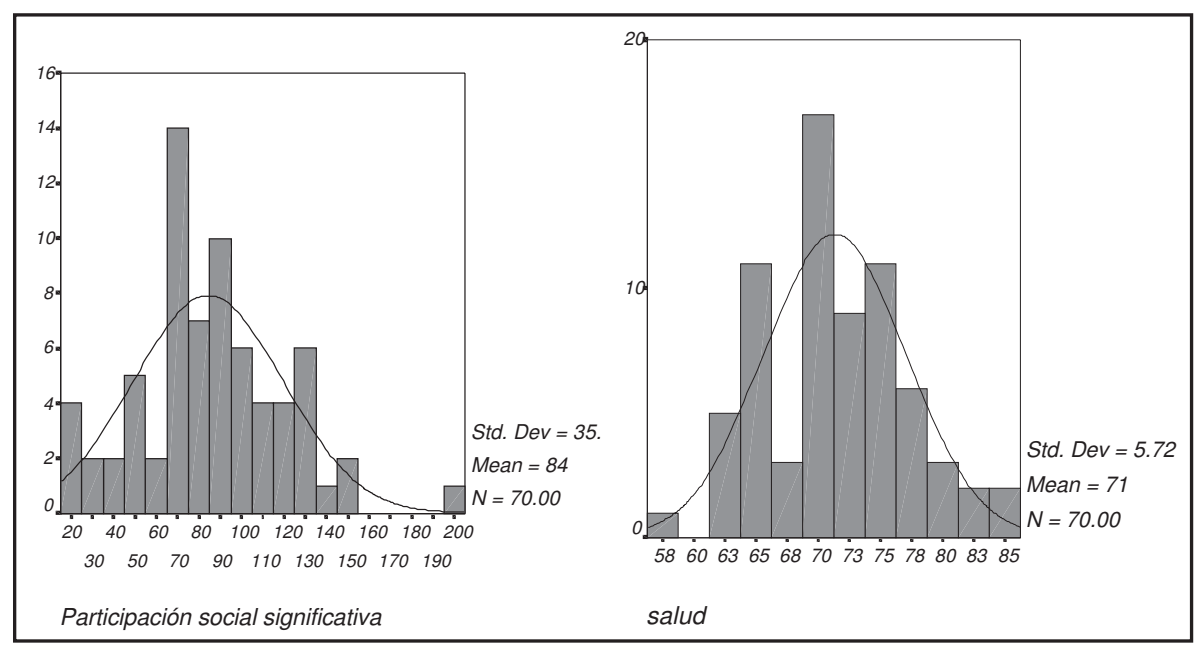

GRÁFICO 1: Histogramas de frecuencias para las variables participación social significativa y salud en un grupo de mujeres ancianas

TABLA 1 - Estadísticos principales para las variables participación

\begin{tabular}{ccccccc}
\hline & MEDIA & MEDIANA & MODA & DESVIO & MIN & MAX \\
& & & & & & \\
\hline PSS & 84.40 & 82.00 & 87.00 & 35.16 & 15 & 199 \\
\hline \multirow{2}{*}{ SALUD } & 71.32 & 71.00 & 71.00 & 5.71 & 58 & 86 \\
\hline
\end{tabular}

Según los resultados arrojados por la pruebas estadísticas utilizadas para conocer la correlación entre las variables en estudio -r de Pearson-, el nivel de PSS se relacionó significativamente con el estado de salud $(\mathrm{r}=$ $0.633, p=0.01$ ). Por lo tanto, podemos decir que existe una correlación positiva entre la PSS y la salud de adultas mayores. En otros términos, a medida que la PSS de la mujer anciana aumenta, se incrementa asimismo su estado de salud (Gráfico 2). 


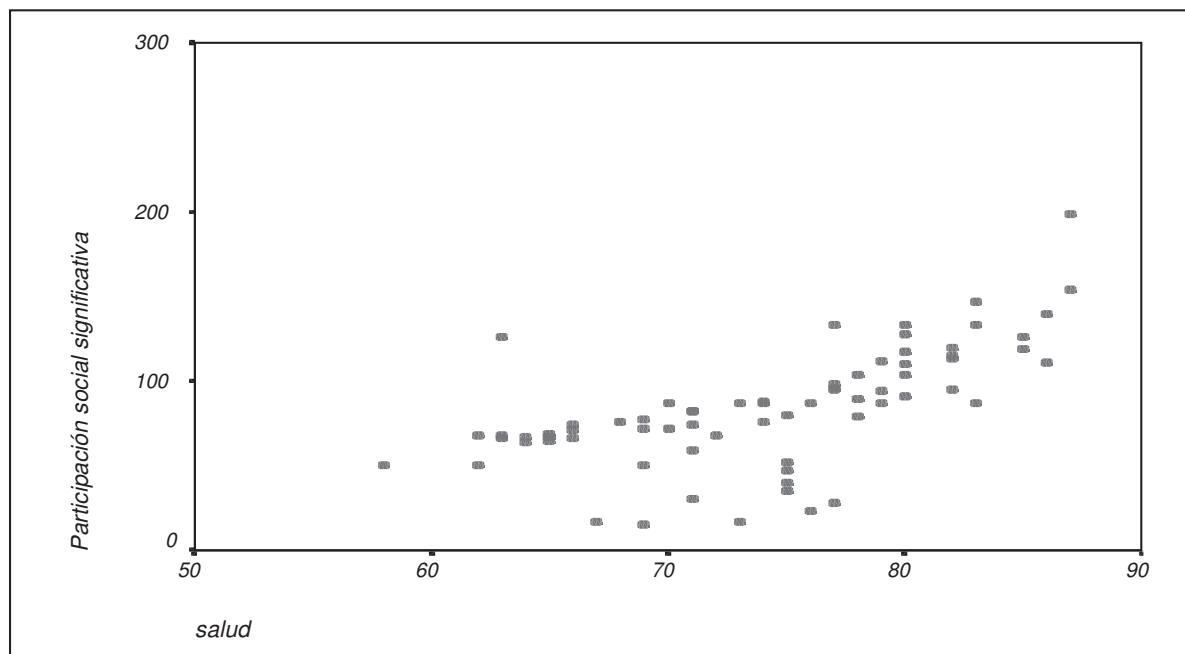

GRÁFICO 2: Gráfico de dispersión de la correlación positiva entre las variables participación social significativa y salud

A partir del test de Kolmogorov-Smirnov se comprobó que la distribución empírica se ajusta a una distribución normal en ambas variables de estudio (K$\mathrm{Z}: 0.677, \mathrm{p}=0.01$ para PSS; K-Z: 0.669, $\mathrm{p}=0.01$ para salud). Esta prueba permite, a partir del comportamiento de estas variables en nuestra muestra, inferir que dicha correlación existe a nivel de la población total.

\section{Discusión}

Los resultados obtenidos muestran una correlación positiva y significativa entre participación en actividades desarrolladas en el seno de relaciones sociales y la salud en las adultas mayores que conformaron nuestra muestra. Por tanto, la participación social y vínculos significativos percibidos por las mujeres de edad avanzada como beneficiosos, serían factores intervinientes en el mantenimiento de la salud. En este estudio se destaca la influencia sobre el estado de salud que tienen los factores sociales ligados a la participación social y relaciones sociales próximas, independientemente de la edad. Estos resultados obtenidos son congruentes con los mencionados estudios empíricos en el campo de la psicogerontología que acuerdan en destacar la relevancia de los factores relativos al ambiente social sobre la salud en la vejez.

La construcción de espacios de participación social significativa (EPSS) para una vejez sana resulta de la confluencia de la valoración social y 
subjetiva de actividades y relaciones sociales, de manera que éstas resulten significativas para el viejo. Por lo tanto, una actividad significativa, según creemos, debe reunir una serie de características para tener efectos en el campo de la salud:

a) realizar la actividad resulta satisfactorio e implica un esfuerzo en términos de ruptura con la rutina propia de la cotidianeidad;

b) durante la misma se producen intercambios entre los participantes que implican un conocimiento de aspectos personales y relaciones próximas;

c) concurrencia de un numero significativo de personas;

d) ámbito extrafamiliar;

e) reconocimiento concreto por parte una autoridad, destinatario, comunidad, etc. en forma de: retribución económica, certificación, agradecimiento explícito, etc.

En función de estas condiciones y de una adecuada oferta de actividades desde lo social, consideramos que, por un lado, se habilita socialmente un espacio simbólico para la vejez; y por otro, esta participación social conlleva cambios en el estilo de vida, el cual en tanto componente del campo de la salud, propiciaría altos índices de salud (según nuestros datos empíricos). A su vez, estos factores inspiran cambios en la representación social de la vejez, que contribuyen a retroalimentar un modelo de envejecimiento participativo, como se observa en la figura 4. 


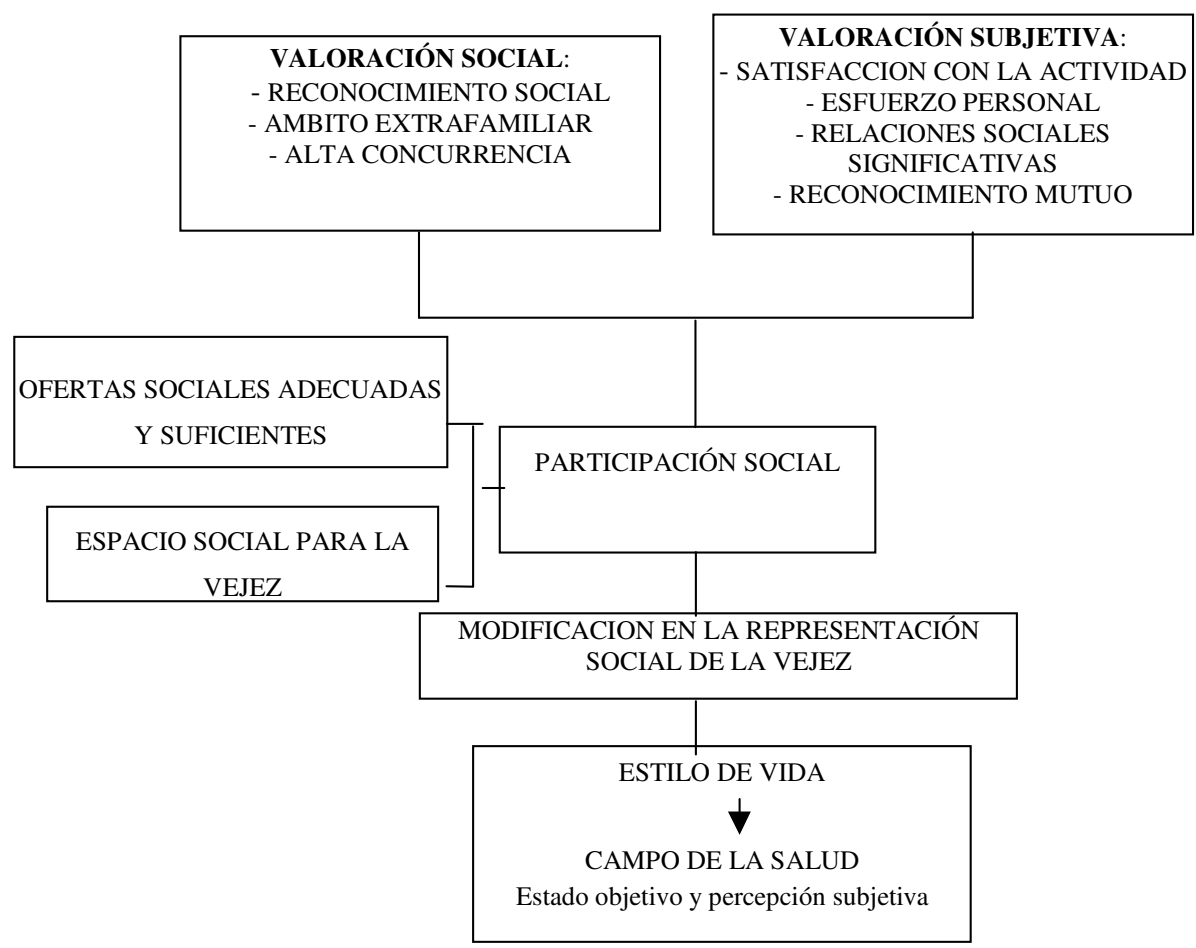

FIGURA 4: Modelo de relación entre participación social significativa y salud en la vejez

A partir de estas ideas, creemos que resulta primordial la diagramación de programas de intervención en salud que favorezca la participación de las personas de edad como actores sociales, asumiendo un rol protagónico en su medio social.

Por otro lado, si bien dada la naturaleza de los estudios en sujetos humanos, la variabilidad sistemática de la variable en estudio salud puede estar afectada por múltiples factores, el diseño de investigación correlacional resultó adecuado para describir las variaciones registradas en una variable en relación a las ocurridas en la otra. No obstante, no se pretende hacer inferencias causales de los datos. Es preciso señalar como limitación de este estudio que al tratarse de una muestra no probabilística, la inferencia de los resultados se restringe al grupo de sujetos considerados. También es oportuno la reproducción futura de este estudio sobre sujetos masculinos para obtener mayor evidencia empírica del alcance de nuestros resultados que destacan la relación entre participación social y salud en la vejez. 


\section{Conclusión}

La salud resulta ser una preocupación relevante en la definición del bienestar de la adulta mayor. Especialmente, la importancia que adquiere la autopercepción de la propia salud merece ser considerada a la hora de definir los estándares de salud en la vejez femenina.

Asimismo, la participación social no supone sólo el desarrollo de actividades, sino el hecho de establecer vínculos en el interior de las mismas percibidos subjetivamente como beneficiosos. En este sentido, considerando a la participación social como un valor propio del campo de la salud, el concepto de salud en la tercera edad se enriquece con la inclusión no sólo de la presencia de estados orgánicos, psíquicos y sociales de bienestar, sino también con la existencia de relaciones sociales significativas.

La participación social significativa permite el desarrollo del potencial personal y los recursos disponibles de las personas de edad, adoptando un estilo de vida activo y modificando aún el medio relacional hacia una renovación de la representación social de la vejez. La pertenencia a un grupo social, donde se mantengan vínculos sociales significativos y se desarrollen actividades valoradas personal y socialmente, resulta eficaz para contrarrestar la errónea asociación vejez y enfermedad, consecuencia del prejuicio social a la vejez.

En definitiva, la construcción de espacios de participación social significativa definidos por relaciones sociales desarrolladas en actividades valoradas social y subjetivamente, favorece un estilo de vida activo y un modelo de envejecimiento que promueve la salud.

Por último, en relación a las ideas precedentes y de acuerdo a nuestros datos empíricos, sería deseable que las políticas sanitarias y los profesionales de la salud que trabajamos en ellas arbitremos programas de promoción de la salud en la vejez - particularmente de la mujer que envejece - que propongan una oferta adecuada de espacios de participación social, contemplando la necesidad de integración social de los adultos mayores, ya que la salud de las personas de edad depende, en forma directa o indirecta, del estilo de vida y de la participación que desarrollen en su medio social. 


\title{
SOCIAL PARTICIPATION SPACES AND HEALTH IN THE FEMALE OLD AGE
}

\begin{abstract}
The current paper proposes to study the correlation between social participation and health in the female old age. By means of a descriptive correlational and cross-sectorial design, it was administrated a structured questionnaire in the frame of a semi-structured interview to a sample of 70 subjects of feminine sex from 60 to 80 years old from Mar del Plata city. It was evaluated the physical and mental health condition and the participation in activities and social relationship socially and subjectively valued. It was considered the frequency of attendance, the satisfaction and the subjective valuation about social participation. We propose the concept of significative social participation spaces (SSPS) which integrates the institutional offer, socially and subjectively valued activities, and significative social relationship, as interdependent components. A quantitative analysis of data was done. The results showed an stadistical significant and positive correlation between significative social participation and health in old women $(r=0.633, p=$ 0.01). Old women who do not participate in activities and social relationship evidence a health condition significantly smaller than those who show an socially active lifestyle. The significative social participation promote an healthful aging model.
\end{abstract}

Keywords: Female Old Age. Significative Social Participation. Health.

\section{REFERÊNCIAS}

BARO, F. Factores psicosociales y la salud de los ancianos. In: Hacia el Bienestar de los Ancianos. Washington: OPS, 1985. P. 87-97. (Pub. cient., 492)

BARROS LAZAETA, C. Aspectos sociales del envejecimiento. Epas: educacion para el autocuidado en salud, Santiago de Chile, p. 57-66, 1989.

BELLO JUJÁN, L.; SUÁREZ RIVERO, J.; PRIETO RAMOS, F.; SERRA MAJEM, L. Variables sociales y sanitarias asociadas a la autopercepción del estado de salud en la población adulta de Gran Canaria. Atención Primaria, Barcelona, v. 24, n. 9, p. 533-536, 1999. 
BERKMAN L. F.; SYME S. L. Social Networks Host Resistance and Mortalility: a nine year follow-op study of Alameda Country Residents. American Journal of Epidemiology, Baltimore, n. 109, p. 186-204, 1979.

BERKMAN, L. F. Assessing the Health Effects of Social Networks and Social Support. Annual Review of Public Health, Palo Alto, v. 5, p. 413-432, May 1984.

BOWLING, A.; BROWNE, P. D. Social Networks, Health and Well-Being Among the Oldest Old in London. Journal of Gerontology, Washington, v. 46, n. 1 , p. $520-532,1991$.

BROADHEAD, W.;KAPLAN, B. H.; JAMES, S. A. et al. The Epidemiologic Evidence for a Relationship Between Social Support and Health. American Journal of Epidemiology, Washington, n. 117, p. 521-537, 1983.

CASSEL, J. Psychology Processes and Stress: theoretical formulations. International Journal of Health Services, Oxford, v. 6, p. 471-482, 1974.

CISAP-2 Definida. Clasificación Internacional de problemas de salud. Atención Primaria. Barcelona: Masson Editores, 1996.

COMITÉ DE CLASIFICACIÓN DE LA WORLD FAMILIY DOCTORS CARING FOR PEOPLE - WONCA. Clasificación Internacional sobre Atención Primaria. CISAP-2, segunda edición, Gran Bretaña: Oxford University, 1998.

CUMMINGS, E.; HENRY, W. E. Growing Old: the process of disengagement. Nueva York: Basic Books, 1961.

DABAS, E. Red de redes: Las prácticas de la intervención en redes sociales. Buenos Aires: Paidos, 1993.

DULCEY-RUIZ, E. La gerontología en la perspectiva de la psicología de la salud. Revista Medicina de la Tercera Edad, Buenos Aires, v. 7, n. 8, p. 9-18, 1988.

EPP, J.; KICKBUSCH, I.; LALONDE, M.; TERRIS, M. Promoción de la salud, una antología. Buenos Aires: OPS, 1996. (Pub. cient., 557).

FERNÁNDEZ-BALLESTEROS, R. et al. Evaluación e intervención psicológica en la vejez. In:__. Biblioteca de Psicología, Psiquiatría y Salud. Barcelona: Martínez Roca, 1992. P. 176-194.

HAREL, Z.; DEIMLONG, G. Social Resources and Mental Health: an empirical refinement. Journal of Gerontology, Washington, v. 39, p. 747-752, 1984. 
HAVIGHURST, R. J. Successful Aging. In: WILLIAMS, R.; TIBBITS, C.; DONOHUE, W. Process of Aging: social and psychological perspectives. Nueva York: Alberton, 1963. P. 299-320.

LALONDE, M. Carta de Ottawa para la promoción de la salud. In: CONFERENCIA SOBRE PROMOCIÓN DE LA SALUD, 1986, Ottawa. Anales... Ottawa: Ministerio de Salud y Bienestar Nacional, 1986. P. 3-5.

LA ROSA, E. Nociones de salud y enfermedad. Revista Médica de la Tercera Edad, Buenos Aires, v. 7, n. 8, p. 5-8, 1988.

LOWENTHAL, M.; ROBINSON, B. Social Networks and Isolations. In: BINSTOCK, R.; SHANAS (Ed.). Handbook of Aging and the Social Sciences. New York: Van Nostrand Reinhold, 1976. P. 432-456.

MATEOS ALVAREZ, R.; CAMPORRO ROCES, B.; BLANCO, J.; PARAMO FERNÁNDEZ, N.; CAROLLO LIMERES, M. C.; RODRÍGUEZ LÓPEZ, A. El apoyo social de los viejos gallegos: resumen de resultados en el marco de un estudio epidemiológico comunitario de salud mental. Anales de Psiquiatría, Madrid, v. 17, n. 5, p. 214, 2001.

MOOS, R. H.; LEMBE, S. Specialized Living Environments for Older People. In: BIRREN, J. E.; SCHAIE, K. W. (Ed.). Handbook of Psychology of ging. 2. ed. Nueva York: Van Nostrand Reinhold, 1985. P. 864-889.

MUCHINIK, E. Las redes sociales de apoyo. Revista Argentina de Clínica Psicológica, Buenos Aires, v. 2, n. 2, p. 174-181, 1990.

NACIONES UNIDAS. Asamblea Internacional sobre el Envejecimiento. Una sociedad para todas las edades. Viena, 1982.

ODDONE, M. J. Dimensiones de la vejez en la sociedad argentina. Buenos Aires: Centro Editor de América Latina, 1990. P. 4-9.

ORGANIZACIÓN MUNDIAL DE LA SALUD. Carta de Ottawa para la promoción de la salud: salud para todos. Revista de Sanidad e Higiene Publica, Madrid, n. 61, p. 129-133, 1986.

Grupo WHOQOL, División de Salud Mental. Instrumento de evaluación de la calidad de vida y la salud. Development of the World Health Organization WHOQOL-BRIEF Quality of Life Assesment. Psychological Medicine, Cambridge, v. 28, p. 551-558, 1998. 
. WHO Basic Documents: constitution of the World Health Organization: Génova, 1948.

PÉREZ-ANDRÉS, C. Mujer, derechos humanos y salud. Revista Española de Salud Pública, Madrid, v. 72, n 2, p. 87-89, 1993.

PINEAULT, R.; DAVELUY, C. La planificación sanitaria: conceptos, métodos y estrategias. Masson: Barcelona, 1995.

PSZEMIAROWER, S. Prevención en Gerontología. Revista Medicina de la Tercera Edad, Buenos Aires, v. 8, n. 7, 1988.

SEIDMANN, S. Soledad, redes sociales de apoyo y estilos de afrontamiento en diferentes grupos generacionales de mujeres. In: REUNIÓN NACIONAL DE LA ASOCIACIÓN ARGENTINA DE CIENCIAS DEL COMPORTAMIENTO, 8., 2001, Rosario, Anales...Rosario: International Union of Psychological Science, 2001. P. $14-16$

THIED AGE LEARNING INTERNATIONAL STUDIES - TALIS. Toulouse: Universite de Toulouse, v. 8, p. 14-18, 85-88, 1998.

THONSE, J. Hacia el bienestar de los ancianos. Buenos Aires: OPS, 1985. (Pub. cient., 492).

WARD, I. A. Informal Networks and Well-Being un Late life: a research agenda. Gerontologist, Washington, v. 25, p. 55-61, 1985. 